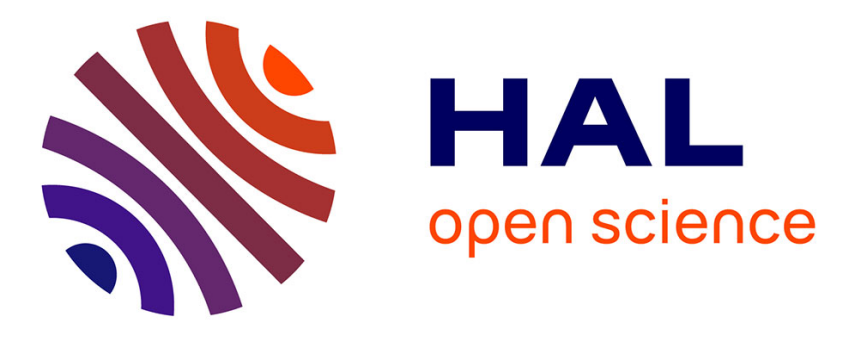

\title{
Mechanical and control-oriented design of a monolithic piezoelectric microgripper using a new topological optimisation method.
}

Mathieu Grossard, Christine Rotinat-Libersa, Nicolas Chaillet, Mehdi Boukallel

\section{To cite this version:}

Mathieu Grossard, Christine Rotinat-Libersa, Nicolas Chaillet, Mehdi Boukallel. Mechanical and control-oriented design of a monolithic piezoelectric microgripper using a new topological optimisation method.. IEEE/ASME Transactions on Mechatronics, 2009, 14 (1), pp.32-45. 10.1109/TMECH.2008.2003437 . hal-00417886

\section{HAL Id: hal-00417886 \\ https://hal.science/hal-00417886}

Submitted on 17 Sep 2009

HAL is a multi-disciplinary open access archive for the deposit and dissemination of scientific research documents, whether they are published or not. The documents may come from teaching and research institutions in France or abroad, or from public or private research centers.
L'archive ouverte pluridisciplinaire HAL, est destinée au dépôt et à la diffusion de documents scientifiques de niveau recherche, publiés ou non, émanant des établissements d'enseignement et de recherche français ou étrangers, des laboratoires publics ou privés. 


\title{
Mechanical and Control-Oriented Design of a Monolithic Piezoelectric Microgripper using a New Topological Optimisation Method
}

\author{
Mathieu Grossard, Christine Rotinat-Libersa, Nicolas Chaillet and Mehdi Boukallel
}

\begin{abstract}
This paper presents a new method developed for the optimal design of piezoactive compliant micromechanisms. It is based on a flexible building block method, called FlexIn, which uses an evolutionary approach, to optimize a truss-like planar structure made of passive and active building blocks, made of piezoelectric material. An electromechanical approach, based on a mixed finite element formulation, is used to establish the model of the active piezoelectric blocks. From the first design step, in addition to conventional mechanical criteria, innovative control-based metrics can be considered in the optimization procedure to fit the open-loop frequency response of the synthesized mechanisms. In particular, these criteria have been drawn here to optimize modal controllability and observability of the system, which is particularly interesting when considering control of flexible structures. Then, a planar monolithic compliant micro-actuator has been synthesized using FlexIn and prototyped. Finally, simulations and experimental tests of the FlexIn optimally synthetized device demonstrate the interests of the proposed optimization method for the design of micro-actuators, microrobots, and more generally for adaptronic structures.
\end{abstract}

Index Terms-Actuator design, balanced gramian, compliant mechanisms, controllability, microgripper, microrobotics, observability, piezoelectricity, topology optimization, vibrations control.

\section{INTRODUCTION}

I $\mathrm{N}$ many applications including Micro Electro Mechanical Systems (MEMS) [1], [2], [3], smart structures [4], [5], surgical tools [6], [7], etc, compliant mechanisms have already been used. They are single-body, elastic continua flexible structures, that deliver the desired motion by undergoing elastic deformation, as opposed to jointed rigid body motions of conventional mechanisms. When considering small scale systems (e.g. for microrobotics use), there are many advantages of compliant mechanisms, among them: simplified manufacturing, reduced assembly costs, reduced kinematic

Manuscript received November 16th, 2007.

M. Grossard is with both the Laboratoire d'Automatique de Besançon (LAB CNRS / ENSMM / UFC), Besançon, F-25000 France, and CEA LIST, Interactive Robotics Unit, Fontenay-aux-Roses, F-92265 France.

C. Rotinat-Libersa and M. Boukallel are with the CEA LIST, Interactive Robotics Unit, Fontenay-aux-Roses, F-92265 France.

$\mathrm{N}$. Chaillet is with the Laboratoire d'Automatique de Besançon (LAB CNRS / ENSMM / UFC), Besançon, F-25000 France.

Corresponding author: Mathieu Grossard, 24 rue Alain Savary, Besançon,

F-25000 FRANCE. E-mail: mathieu.grossard@ens2m.fr

phone: + 33381402806 , fax: + 33381402809

Paper type: regular paper.

Technical area: Robotics. noise, no wear, no backlash, high precision, and ability to accommodate unconventional actuation schemes.

One type of smart material-based actuator typically used to actuate compliant structures is piezoceramic PZT actuators: when compared to other conventional actuation principles at small scales, they have very appealing properties in the sense of micromechatronic design. When integrated inside a compliant mechanism, piezoelectric actuators can exert actuation forces to the host structure without any external support. They can also be manufactured into the desired shape, while making realistic the realization of piezoelectric monolithic compliant mechanisms, such as microgrippers [8]. Piezoelectric actuation is mostly used for microrobot design in order to achieve nanometric resolutions, and has naturally became widespread in micromanipulation systems [9].

However, one limitation of piezoelectric actuators is that they are capable of producing only about $0.1 \%$ strain, resulting in a restricted range of motion. A number of papers only address the problem of optimally designing coupling structures to act as stroke amplifiers of the piezoelectric actuator [10], [11], [12]. Opposite to these methods, where the piezoelectric elements in the structure are predetermined, a large body of work related to optimization of active structures deals with the optimal location of actuators on a given structure [13]. Another general approach to optimally design actuated structures is to simultaneously [14] or separately [15] optimize the actuator size. Finally, few studies consider the topology optimization (shape) of monolithic PZT active structures [16].

Moreover, some meso-scale robots exploit the high bandwidth of piezoelectric actuators (e.g. to achieve locomotion through a stick-slip principle [17]). Often, resulting piezoactuated devices are electromechanically tuned resonating microrobots. But, previous works in topology design of active compliant structures have mainly focused on quasi-static applications, which may be sub-optimal in dynamic operations, or, worse, may induce degraded functioning. Very few related works deal with topological optimization method including frequency response analysis [14], [18]. There, the objective functions generally use the maximization of either geometrical advantage (stroke amplification), or mechanical advantage (force amplification), only in the restrictive case of predetermined harmonic loadings.

To improve such active compliant micromechanisms performances, it can be useful to optimize them from the first 
designing step, taking into account versatile microrobotic criteria [19] . A global systematic design approach is presented in this paper, where topology optimization of the piezoactive structure, as well as that of its frequency response, is used to design compliant smart mechanisms. This method is based on the flexible building block method called FlexIn ("Flexible Innovation"). It considers a planar compliant mechanism as an assembly of both passive and piezoactive compliant building blocks, and uses a multi-objective genetic algorithm to optimize these structures. To complete the panel of purely mechanical criteria, innovative control-based metrics have been newly proposed in FlexIn. These criteria are useful tools to ensure the efficient control of the flexible structures afterwards. Indeed, each FlexIn generated structure is described by its own state model, giving rise to several control-oriented fitnesses. In particular, the different possible interpretations of modal gramians for flexible structures in terms of modal controllability and observability [20] can be taken into account by many criteria in the optimization procedure. They can address several problems, such as avoiding noise amplification and decoupling disturbances [21], [22], avoiding spillover-effects [23], improving dynamic input/output performances [24], [25], [26] which can be of great interest in the control-oriented design of mechatronic devices.

This paper is organized as follows: firstly, we will briefly review the underlying idea of the FlexIn methodology for the optimal design of smart compliant mechanisms. In a second part, the electromechanical approach, based on a mixed finite element formulation, is established for the model of the piezoactive building blocks. Thirdly, the state model approach used in FlexIn is presented, where the mechanism is discretized on its modal components. At this stage, a topology design strategy is drawn to take into account, in the optimization algorithm, accurate model-reduction and control of flexible structures. Two resulting numerical criteria will help meeting open-loop input-output transfer performances with specific operation requirements. In addition to purely static mechanical criteria, these new control-oriented metrics are simultaneously used to optimally synthesize a compliant piezoactuator. In the fifth part, problem specification, optimization results and simulation of the pseudo-optimal piezoactuator are presented. The last part deals with the realisation of the optimally designed piezoactuator prototype, and experimental validation. The results obtained demonstrate the interests of the FlexIn optimization method for the design of unconventional micro-actuators.

\section{FLEXIN: A COMPLIANT MECHANISM STOCHASTIC DESIGN METHODOLOGY}

In this section, we briefly present the flexible building block method, which has been implemented for the optimal design of micromechanical planar mechanisms in a software called FlexIn (developed with Matlab $^{\circledR}$ ). It uses a multiobjective evolutionary algorithm approach for the optimal design of smart compliant mechanisms made of an assembly of elementary passive and active compliant building blocks, chosen in two specific libraries. Detailed descriptions of the method can also be found in [27], [28], [29], [30], [31].

\section{A. Compliant building blocks}

Two libraries of compliant elements in limited number are proposed in FlexIn. These bases are composed respectively of 36 passive and 19 piezoactive block elements, made of beams assembly (Fig.1). They are sufficient to build a high variety of topologies. Moreover, the block feasibility related to fabrication process constraints can also be taken into account at this stage.

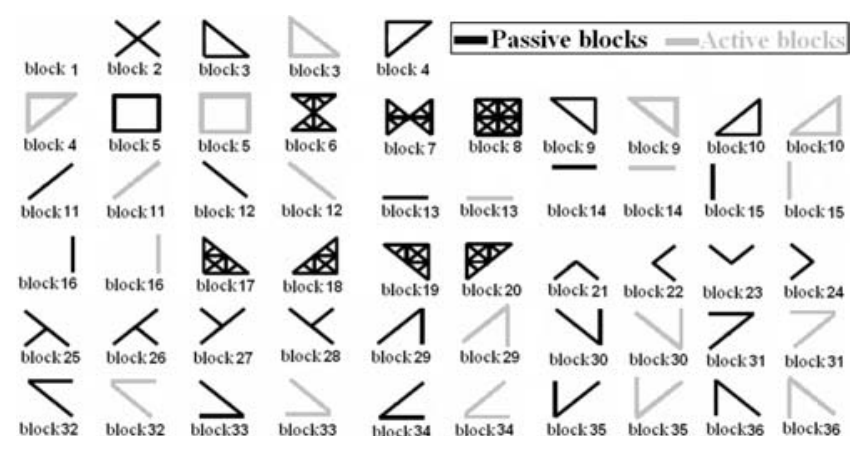

Fig. 1. Passive (black) and piezoactive (grey) libraries of compliant building blocks, for planar compliant mechanisms synthesis using FlexIn.

\section{B. Principles of the method and design parameters}

The purpose of FlexIn is to optimally design realistic compliant structures. The specification of a planar compliant mechanism problem considers specific boundary conditions: fixed frame location, input (actuators), contacts and output (end-effector). Different types of actuation principles can be used: either external or internal force/displacement actuators defined at particular nodes of the mesh [28], or integrated piezoactive elements taken from the active library above [29], [31]. The design method consists of searching for an optimal distribution of allowed passive building blocks, as well as for the optimal set of structural parameters and materials, in order to obtain relevant monolithic compliant structures. The location of fixed nodes, contacts, and that of the actuators and/or piezoactuated blocks can also be considered as optimization parameters. The topology optimization method, inspired from [32], uses a genetic algorithm approach, where structures are considered as individuals in a population(Fig.2). Compliant mechanisms description is made using discrete variable parameterization and considering conception requirements (mesh size, topology, material and thickness, boundary conditions). At every generation, the designed criteria are evaluated for each individual, and a Pareto graph is computed for multiobjective optimization. A tournament selects genitors amongst the whole population, and randomly generates two offsprings by parent pair. The algorithm continues until criteria desired values are reached or algorithm stagnates. 


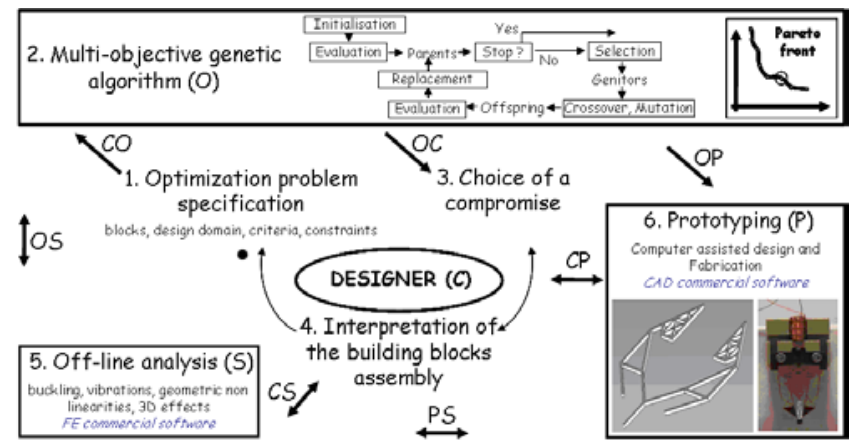

Fig. 2. Flowchart of the FlexIn optimal design method of compliant structures (multicriteria optimization).

\section{Multi-criteria genetic algorithm}

Many fitness functions are available in FlexIn, thus allowing the optimal synthesis of devices within a wide schedule of conditions.

- Several static mechanical fitness can be specified for the optimization problem: free displacement and blocking force at the output port, geometric advantage (GA) i.e. ratio between output and input strokes, mechanical advantage (MA) i.e. ratio between output and input forces, etc.

- Various dynamic control-oriented metrics have been newly implemented in FlexIn to meet specific control requirements for microrobotics devices [26]. Obviously, the design strategy depends on the metrics chosen, which must be based on the real needs for the device use.

Let us note that multi-degrees of freedom mechanism design can also be considered. At convergence step, the optimization algorithm generates a set of pseudo-optimal solutions (see step 2 in Fig.2) in the case of multicriteria optimization (and obviously only one global optimal solution for monocriterion optimization). The designer can next choose, analyse and interpret the obtained structures that best suit his design problem (see 3 and 4 in Fig.2). A finite element (FE) software can be used for subsequent FE analyze and validation of the chosen design solution, for other criteria not considered during the optimization stage (see 5 in Fig.2).

\section{ElECtRo-MECHANiCAL FE MODEL OF THE PIEZOELECTRIC STRUCTURES}

In this section, we present the 2D FE modelling adopted for the active piezoelectric building blocks, and the way these blocks are implemented in the optimization method. In this study, only in-plane forces and displacements are considered.

\section{A. Elementary piezoelectric beam}

In FlexIn, it is assumed that the compliant mechanisms are undergoing structural deformations, mainly due to the inplane bending of the beams constituting the blocks. Thus, the models of the blocks are obtained considering NavierBernoulli beam type finite elements. Structural parameters of each rectangular block are height, width and thickness. Material characteristics of each block are parameterized by
Young's modulus, Poisson's ratio, yield strength, density, and piezoelectric coefficients for the piezoactive blocks. To allow the calculation of different optimization criteria, FlexIn uses the FE model of each block of the libraries. To obtain the FE formulation of the piezoelectric blocks, a model of a piezoelectric beam is first needed.

We consider that the piezoceramic beams constituting the blocks are perfectly bonded to electrodes at their lower and upper faces (Fig.3). Exploiting the transverse effect of piezoelectricity, longitudinal deformation $S_{11}$ along $L$ dimension is generated under the transverse electric field $E_{3}$. Considering the one-dimensional form of piezoelectricity equation along the length direction of the beam, the piezoelectric coupling matrix $\mathbf{d}$ and the stress-free electric permittivity matrix $\varepsilon^{t}$ are each represented by a single coefficient, $d_{31}$ and $\varepsilon_{33}$ respectively, and the electric-free compliance matrix $\mathbf{s}^{E}$ is represented by $s_{11}^{E}$. The subscript " $t$ " denotes the transpose of a matrix. Hence, within the piezoelectric beam, the constitutive relations for the strain $S_{11}$ and electric displacement $D_{3}$, as functions of stress $T_{11}$ and electric field $E_{3}$, take the form [33]:

$$
\left\{\begin{array}{c}
S_{11} \\
D_{3}
\end{array}\right\}=\left[\begin{array}{ll}
s_{11}^{E} & d_{31} \\
d_{31} & \varepsilon_{33}^{T}
\end{array}\right]\left\{\begin{array}{c}
T_{11} \\
E_{3}
\end{array}\right\}
$$

The superscripts " $E$ " and " $T$ " refer to values taken respectively at constant electric and stress fields.

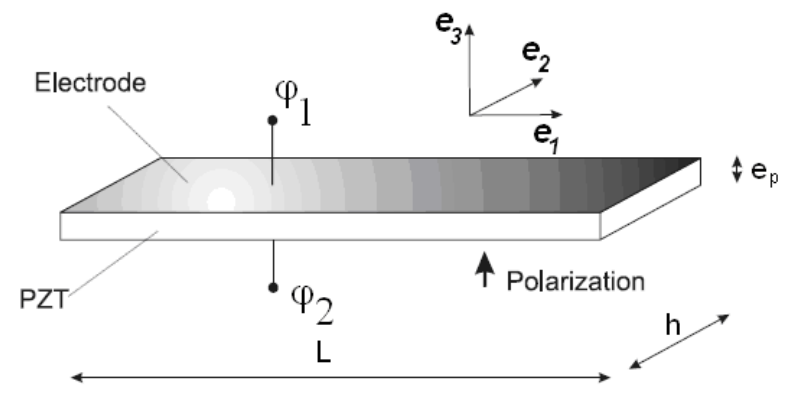

Fig. 3. Thickness-polarized piezoelectric beam transducer with electroded surfaces, and orientation in the material reference frame $\left(\mathbf{e}_{1}, \mathbf{e}_{2}, \mathbf{e}_{3}\right) \cdot \varphi_{1}$ and $\varphi_{2}$ denotes the electric potential of the electrodes.

\section{B. 2D FE formulation of the piezoactuated beam}

The displacement field over a planar beam element is described from its longitudinal $u$, tangential $v$ and rotational $\omega$ components at $x_{p}$ curvilinear abscissa (Fig.4), and is related to the corresponding node values $\eta_{\mathbf{b}}=$ $\left(u_{A}, v_{A}, \omega_{A}, u_{B}, v_{B}, \omega_{B}\right)_{R_{p}}^{t}$ in the beam coordinate system $R_{p}=\left(A, \mathbf{x}_{\mathbf{p}}, \mathbf{y}_{\mathbf{p}}, \mathbf{z}_{\mathbf{p}}\right)$. From Hamilton's principle modified for general electromechanical system [34], [35] the model of the active beam takes the following form:

$$
\mathbf{M}_{\mathbf{b}} \ddot{\eta}_{\mathrm{b}}+\mathbf{K}_{\mathbf{b}} \eta_{\mathbf{b}}=\mathbf{G}_{\mathbf{b}} \boldsymbol{\Phi}_{\mathbf{b}}+\mathbf{F r}_{\mathbf{b}}
$$

where $\mathbf{M}_{\mathbf{b}}, \mathbf{K}_{\mathbf{b}}$ and $\mathbf{G}_{\mathbf{b}}$ are respectively the mass, stiffness and electromechanical coupling beam matrices. $\boldsymbol{\Phi}_{\mathbf{b}}=\left[\varphi_{\mathbf{1}} \varphi_{\mathbf{2}}\right]^{t}$ is the vector representing the electric potentials on the upper and lower faces of the piezoelectric beam. Matrix $\mathbf{G}_{\mathbf{b}}$ in (2) induces piezoelectric loads, which makes the actuator beam 
expand (or contract) proportionally to the external controlled potential difference $\left(\varphi_{\mathbf{1}}-\varphi_{\mathbf{2}}\right)$. The forces vector $\mathbf{F r}_{\mathbf{b}}$, is due to the variational mechanical work terms, and is written $\mathbf{F r}_{\mathbf{b}}=$

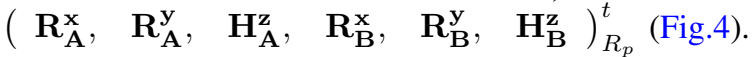

Displacement field is related to the corresponding node values $\eta_{\mathbf{b}}$ by the mean of the shape functions, calculated under Euler-Bernoulli beam assumptions. Detailed derivations can be readily found in finite element textbooks. Corresponding matrices in (2) are simply expressed as follows:

$$
\begin{aligned}
& \mathbf{M}_{\mathbf{b}}=\rho A\left(\begin{array}{cccccc}
\frac{L}{3} & 0 & 0 & \frac{L}{6} & 0 & 0 \\
& \frac{13 L}{35} & \frac{11 L^{2}}{210} & 0 & \frac{9 L}{70} & -\frac{13 L^{2}}{420} \\
& & \frac{L^{3}}{105} & 0 & \frac{13 L^{2}}{420} & -\frac{L^{3}}{140} \\
& & & \frac{L}{3} & 0 & 0 \\
& (\text { sym. }) & & & \frac{13 L}{35} & -\frac{11 L^{2}}{210} \\
& & & & & \frac{L^{3}}{105}
\end{array}\right)_{R_{p}} \\
& \mathbf{K}_{\mathbf{b}}=Y\left(\begin{array}{cccccc}
\frac{A}{L} & 0 & 0 & -\frac{A}{L} & 0 & 0 \\
& \frac{12 I}{L^{3}} & \frac{6 I}{L^{2}} & 0 & -\frac{12 I}{L^{3}} & \frac{6 I}{L^{2}} \\
& & \frac{4 I}{L} & 0 & -\frac{6 I}{L^{2}} & \frac{2 I}{L} \\
& & & \frac{A}{L} & 0 & 0 \\
& (\text { sym. }) & & & \frac{12 I}{L^{3}} & -\frac{6 I}{L^{2}} \\
& & & & & \frac{4 I}{L}
\end{array}\right)_{R_{p}} \\
& \mathbf{G}_{\mathbf{b}}=Y h d_{31}\left(\begin{array}{cccccc}
1 & 0 & 0 & -1 & 0 & 0 \\
-1 & 0 & 0 & 1 & 0 & 0
\end{array}\right)_{R_{p}}^{t}
\end{aligned}
$$

where $I=\frac{e_{p} h^{3}}{12}$ designates the inertia moment of the beam cross section $A=e_{p} h, \rho$ the density of the beam and $Y$ its Young's modulus. The latter is defined by: $Y=1 / s_{11}$.

Some particular piezoelectric materials (e.g. the crystallographic class $6 \mathrm{~mm})$ are isotropic in $(0, \mathbf{x}, \mathbf{y})$ plane. Thus, mechanical and piezoelectric beam characteristics are invariant by in-plane rotation $\theta$ around $z$-axis (Fig.4), so that (2) is the general 2D FE model for all the beams constituting a planar truss whatever its orientation is.

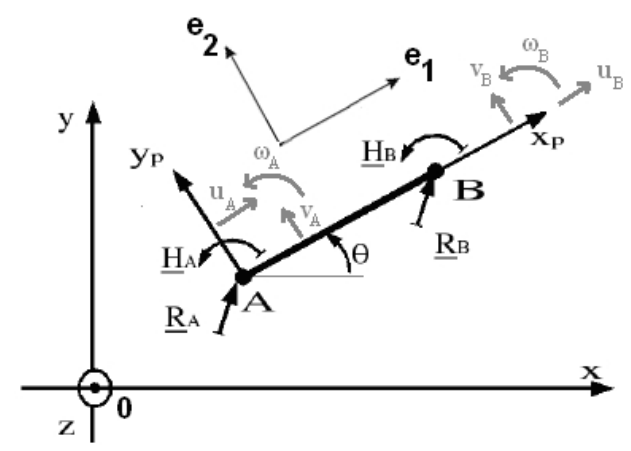

Fig. 4. Curvilinear coordinates of the piezoelectric beam $A-B$, and its orientation in the global coordinate system $R^{\prime}=(0, \mathbf{x}, \mathbf{y}, \mathbf{z}) . \mathbf{R}$ and $\mathbf{H}$ represent the in-plane nodal force and moment at the beam extremities.

\section{Calculation of the active block FE model matrices}

As mentioned in section II.A., a library of active compliant elements made of elementary piezoelectric beams has been implemented in FlexIn. The active blocks present some various topologies, as shown in (Fig.1) and (Fig.5). Their advantage is that they can furnish multiple coupled degrees of freedom (dofs), thus generating more complex movements with only one building block. As for passive blocks, the mass $\mathbf{M}_{\mathbf{B}}$, stiffness $\mathbf{K}_{\mathbf{B}}$ and electromechanical coupling $\mathbf{G}_{\mathbf{B}}$ matrices of each piezoactuated block are obtained by the assembly of mass $\mathbf{M}_{\mathbf{b}}^{\prime}$, stiffness $\mathbf{K}_{\mathbf{b}}^{\prime}$ and electromechanical coupling $\mathbf{G}_{\mathrm{b}}^{\prime}$ matrices of beams, which are expressed in the global coordinate system $R^{\prime}=(0, \mathbf{x}, \mathbf{y}, \mathbf{z})$. The last matrices are simply deduced from $\mathbf{M}_{\mathbf{b}}, \mathbf{K}_{\mathbf{b}}$ and $\mathbf{G}_{\mathbf{b}}$ by the base change formula, accounting for the in-plane $\theta$-rotation transformation.

The results obtained by our $2 \mathrm{D}$ active block FE model have been validated in static conditions and under various boundary conditions using a commercial 3D multiphysics finite element software [31].

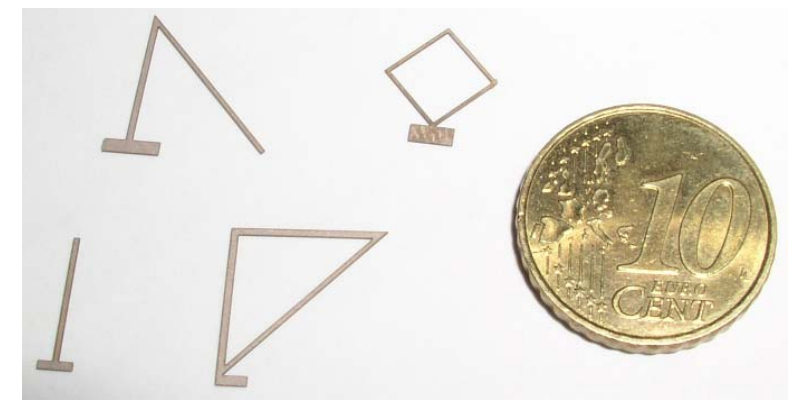

Fig. 5. Examples of monolithic PIC151 piezoelectric blocks (thickness $e_{p}=$ $200 \mu \mathrm{m})$, machined by a laser cutting process.

\section{FE model of piezoeletric structures}

The mass, stiffness and electromechanical coupling matrices of each block are calculated numerically, considering every combination of the discrete values allowed for the structural optimization variables, i.e. material and size of the blocks. Thus, the calculation of the different matrices of each valuedblock is done one time only at the beginning of the optimal design problem (before running the genetic algorithm), which saves running time.

During the optimization, candidate structures are generated by the genetic algorithm. The conservative dynamic behaviour of a structure is described through its mass $\mathbf{M}_{\mathrm{g}}$, stiffness $\mathbf{K}_{\mathrm{g}}$ and electromechanical coupling $\mathbf{G}_{\mathbf{g}}$ matrices, obtained by the assembly in $R^{\prime}$ of the matrices of all the blocks constituting the structure, as follows:

$$
\mathbf{M}_{\mathrm{g}} \ddot{\eta}_{\mathrm{g}}+\mathbf{K}_{\mathrm{g}} \eta_{\mathrm{g}}=\mathbf{G}_{\mathrm{g}} \boldsymbol{\Phi}_{\mathrm{g}}+\mathbf{F r}_{\mathrm{g}}
$$

This assembly is done during the optimisation process at each generation and for each individual. In (6), $\eta_{\mathrm{g}}$ refers to the nodal displacements of the truss-structure, $\boldsymbol{\Phi}_{\mathrm{g}}$ to the external voltages applied to the upper and lower electrodes of each block constituting the structure (in case of piezoactuation) and $\mathbf{F r}_{\mathrm{g}}$ to the external mechanical nodal forces applied to the structure. 


\section{USEFUL MEASURES FOR EVALUATION OF INPUT-OUTPUT TRANSFER PERFORMANCES OF FLEXIBLE SYSTEMS}

From the computation of the linear state model of compliant systems, an optimal topology design strategy is derived taking into account control considerations. New FlexIn numerical criteria help reaching input-output transfer performances with specific operation requirements. In particular, coefficients of the modal transfer expansion of the system, as well as the joint modal controllability and observability degrees, are useful ways to characterize the open-loop system behaviour.

\section{A. New criteria for control-oriented design of compliant struc- tures with FlexIn}

The two significant design tasks in flexible structure control are the identification of the dominant modes to build an appropriate reduced model, and the control strategy design.

1) Reduced model: Since the dynamic model of a flexible structure is characterized by a large number of resonant modes, accurate identification of all the dominant system dynamics often leads to very high order model. Thus, a model reduction is required. A number of approaches for model reduction have been developed, such as model reduction via balanced realization [36]. Since the approaches are generally based on the order reduction of a finite dimensional model, there is a limit on how many high frequencies can be included.

Thus, in FlexIn, a first criterion has been drawn to optimize the reduced-model accuracy of the systems, while limiting spillover effects. Given a set of structures to optimize, the optimal structures are chosen as the ones guaranteeing the highest joint controllability and observability for all the modes in the bandwidth of interest, while providing the minimum joint controllability and observability of the neglected modes. This criterion will enable the rise of structures with accurate reduced model, based on a few highly dominant modes, allowing the easy identification and computation of state model, well adapted to further design and implementation of the control system.

2) A pseudo-collocated active structure: There are a number of difficulties associated with the control of flexible structures (amongst them, variable resonance frequencies and highly resonant dynamics). Traditional control system design techniques such as $L Q G, H_{2}$ and $H_{\infty}$ commonly appear in research works ( [37], [38], [39]). But, the performances of such high authority controllers appear to be very sensitive to model uncertainty and modeling errors introduced by model truncation. Moreover, the direct use of such advanced techniques can have the disadvantages to produce control systems of high order and/or poor robustness, associated with poor dynamic performance sometimes.

Nevertheless, for some specific class of flexible structures, which can be modeled as collocated resonant systems, active damping controllers (PPF, IFF,...) have proven to offer greater robustness, performance, and ease of implementation relatively to traditional techniques. They are often focused on damping the dominant modes [40]. Although the flexible structures rarely present natural collocated designs, an other optimization criterion, based on the modal expansion of SISO systems transfer function, has been established in FlexIn to force the structures to have an actuator/sensor collocated behaviour in terms of frequency response function.

These two new criteria, when used simultaneously, provide a great deal of information concerning the closed-loop device performances that are achievable with this particular open-loop frequency configurations.

\section{B. Modal equations of motion of flexible structures}

As in [41], each flexible structure synthesized by FlexIn is defined as a finite-dimension, controllable and observable linear system with small damping and complex conjugate poles. Its undamped dynamic behaviour is modeled by the following second-order differential matrix equations:

$$
\begin{aligned}
& \mathbf{M}_{\mathbf{g}} \ddot{\eta}_{\mathrm{g}}+\mathbf{K}_{\mathrm{g}} \eta_{\mathrm{g}}=\mathbf{E}_{\mathbf{g}} \mathbf{u} \\
& \mathbf{y}=\mathbf{F}_{\mathbf{g}} \eta_{\mathrm{g}}
\end{aligned}
$$

Let us consider in the following the integers $p, s$, and $r$, which denote the numbers of degrees of freedom (DOF) of the structure, inputs (i.e. actuators) and observed outputs (sensors), respectively. In (7), remind that $\eta_{\mathbf{g}}$ is the $p \times 1$ nodal displacement vector as defined previously. $\mathbf{u}$ is the $s \times 1$ input vector which defines the controlled command of the actuator. For example, in case of a piezoelectric actuation scheme, $\mathbf{u}$ is defined by $\boldsymbol{\Phi}_{\mathbf{g}}$. In that case, the $p \times s$ input matrix $\mathbf{E}_{\mathbf{g}}$ is exactly $\mathbf{G}_{\mathbf{g}} \cdot \mathbf{y}$ is the $r \times 1$ output vector, defined from the $r \times p$ output displacement matrix $\mathbf{F}_{\mathbf{g}}$. Each element of $\mathbf{u}$ (resp. y) denotes a physical actuator (resp. sensor) whose related DOF is defined by the location of the nonzero entry in the corresponding column in $\mathbf{E}_{\mathbf{g}}$ (resp. row in $\mathbf{F}_{\mathbf{g}}$ ).

By means of modal decomposition, a solution of the form

$$
\eta_{\mathbf{g}}(t)=\sum_{i=1}^{p} \boldsymbol{\Psi}_{\mathbf{i}} \mathbf{q}(t)=\Psi_{\mathbf{q}}(t)
$$

is considered, which consists of a linear combination of mode shapes $\Psi_{\mathbf{i}} . \mathbf{q}$ is the $p \times 1$ modal displacement vector. The eigenvectors matrix $\Psi=\left[\begin{array}{lll}\Psi_{1} & \ldots & \Psi_{p}\end{array}\right]$ and corresponding eigenfrequencies $\omega_{i}$ are obtained as solutions of the free undamped vibration eigenproblem:

$$
\left(\mathbf{K}_{g}-\omega_{i}^{2} \mathbf{M}_{g}\right) \mathbf{\Psi}_{i}=0
$$

because the damping has very little influence on the natural frequencies of flexible structures synthesized. Eigenfrequencies are conventionally sorted in ascending order $\omega_{1}^{2} \leq \omega_{2}^{2} \leq$ $\ldots \leq \omega_{p}^{2}$, and $\Psi$ is chosen normalized to the mass matrix.

Replacing $\eta_{\mathbf{g}}$ by $\boldsymbol{\Psi}_{\mathbf{q}}$ in (7), multiplying on the left by $\boldsymbol{\Psi}^{t}$, the induced orthogonality relationships in modal form lead to

$$
\begin{aligned}
& \ddot{\mathbf{q}}+\operatorname{diag}\left(\omega_{i}^{2}\right) \mathbf{q}=\boldsymbol{\Psi}^{t} \mathbf{E}_{\mathbf{g}} \mathbf{u} \\
& \mathbf{y}=\mathbf{F}_{\mathbf{g}} \mathbf{\Psi} \mathbf{q}
\end{aligned}
$$

We can now introduce diagonal damping by using Basil's hypothesis, so that the first equation in (10) becomes

$$
\ddot{\mathbf{q}}+\operatorname{diag}\left(2 \xi_{i} \omega_{i}\right) \dot{\mathbf{q}}+\operatorname{diag}\left(\omega_{i}^{2}\right) \mathbf{q}=\Psi^{t} \mathbf{E}_{\mathbf{g}} \mathbf{u}
$$


where $\xi_{i}$ is the $i^{t h}$ modal damping ratio. This hypothesis can be made without loss of generality because, in most cases, the system to control is slightly damped in the low-frequency band, where the modes are well separated. Moreover, the model has to be identified on the real structure, since the nature of damping is not exactly known a priori. A last reason of using Basil's hypothesis in FlexIn is that it is more convenient for the designer to specify the $\xi_{i}$ coefficients in the algorithm procedure.

According to equation (11) in modal space, all equations of motion are now decoupled and are similar to a set of independent $2^{\text {nd }}$ order lightly damped harmonic oscillators.

\section{State model of flexible structures}

One interesting state vector $\mathbf{x}$, of dimension $2 p \times 1$, consists of modal velocities and frequency weighted modal displacements:

$$
\mathbf{x}=\left(\begin{array}{ccccc}
\dot{q}_{1} & \omega_{1} q_{1} & \ldots & \dot{q}_{p} & \omega_{p} q_{p}
\end{array}\right)^{t}
$$

with the advantage that the elements of state vector corresponding to each mode are about the same magnitude. This has computational advantages and has been therefore implemented yet [42]. This yields the matrices triplet $(\mathbf{A}, \mathbf{B}, \mathbf{C})$ which denotes the modal state-space representation of a structure as stated below,

$$
\begin{aligned}
& \dot{\mathbf{x}}=\mathbf{A x}+\mathbf{B u}, \\
& \mathbf{y}=\mathbf{C x} .
\end{aligned}
$$

The matrices take the forms $\mathbf{A}=\operatorname{diag}\left(\begin{array}{lll}\mathbf{A}_{1}, \ldots, & \mathbf{A}_{\mathbf{p}}\end{array}\right)$, $\mathbf{B}=\left(\begin{array}{lll}\mathbf{B}_{\mathbf{1}}^{t}, & \ldots, & \mathbf{B}_{\mathbf{p}}^{t}\end{array}\right)^{t}$, and $\mathbf{C}=\left(\begin{array}{lll}\mathbf{C}_{\mathbf{1}}, & \ldots, & \mathbf{C}_{\mathbf{p}}\end{array}\right)$, with, for $i=1, \ldots, p$,

$$
\begin{gathered}
\mathbf{A}_{\mathbf{i}}=\left[\begin{array}{cc}
-2 \zeta_{i} \omega_{i} & -\omega_{i} \\
\omega_{i} & 0
\end{array}\right], \\
\mathbf{B}_{\mathbf{i}}=\left[\begin{array}{c}
\mathbf{b}_{\mathbf{i}} \\
\mathbf{0}
\end{array}\right], \\
\mathbf{C}_{\mathbf{i}}=\left[\begin{array}{ll}
\mathbf{0} & \frac{\mathbf{c}_{\mathbf{i}}}{\omega_{i}}
\end{array}\right],
\end{gathered}
$$

where $\mathbf{b}_{\mathbf{i}}=\Psi_{\mathbf{i}}^{t} \mathbf{E}_{\mathrm{g}}$ is $1 \times s$ size, and $\mathbf{c}_{\mathbf{i}}=\mathbf{F}_{\mathrm{g}} \boldsymbol{\Psi}_{\mathbf{i}}$ is $r \times 1$ size. $\mathbf{b}_{\mathbf{i}}$ and $\mathbf{c}_{\mathbf{i}}$ are the $i^{t h}$ row of $\Psi^{t} \mathbf{E}_{\mathbf{g}}$ and the $i^{t h}$ column of $\mathbf{F}_{\mathbf{g}} \Psi$ respectively. It is important to note that $\mathbf{A}$ matrix depends on the structure itself (eigenfrequencies and modal damping ratios), $\mathbf{B}$ matrix on the location and class of actuators, and C matrix on location and class of sensors. This modal state is considered to be a physical coordinate because of its direct physical link to structural mode shapes.

As another formulation of (13), the Frequency Responses Functions (FRF) matrix between outputs $\mathbf{y}$ and inputs $\mathbf{u}$ can be viewed as the sum of all the modal contributions, as follows:

$$
\mathbf{y}(j \omega)=\mathbf{G}(j \omega) \mathbf{u}(j \omega)
$$

where the $r \times s$ FRF matrix is

$$
\mathbf{G}(j \omega)=\sum_{i=1}^{p} \mathbf{G}_{\mathbf{i}}(j \omega)=\sum_{i=1}^{p} \frac{\mathbf{c}_{\mathbf{i}} \mathbf{b}_{\mathbf{i}}}{\omega_{i}^{2}-\omega^{2}+2 j \xi_{i} \omega_{i} \omega}
$$

\section{Computation of the controllability and observability grami-} ans

Controllability $\left(\mathbf{W}_{\mathbf{c}}\right)$ and observability $\left(\mathbf{W}_{\mathbf{o}}\right)$ gramians are convenient forms to characterize system controllability and observability. For stable A, they are obtained from algebraic solutions of the following Lyapunov equations:

$$
\begin{aligned}
& \mathbf{A} \mathbf{W}_{\mathbf{c}}+\mathbf{W}_{\mathbf{c}} \mathbf{A}^{t}+\mathbf{B B}^{t}=\mathbf{0} \\
& \mathbf{A}^{t} \mathbf{W}_{\mathbf{o}}+\mathbf{W}_{\mathbf{o}} \mathbf{A}+\mathbf{C}^{t} \mathbf{C}=\mathbf{0}
\end{aligned}
$$

and the energetic interpretations are known [20].

Assuming the damping ratios are infinitely small and the natural frequencies well spaced, the block diagonal forms of $(\mathbf{A}, \mathbf{B}, \mathbf{C})$ triplet can be exploited to give closed-form analytical solutions for the expression of the modal controllability and observability gramians [43]. They are diagonal, and equal to:

$$
\begin{aligned}
& \mathbf{W}_{\mathbf{c}}=\operatorname{diag}\left(\begin{array}{lll}
\mathbf{W}_{\mathbf{c}_{11}}, & \ldots, & \mathbf{W}_{\mathbf{c}_{\mathrm{pp}}}
\end{array}\right) \\
& \mathbf{W}_{\mathbf{o}}=\operatorname{diag}\left(\begin{array}{lll}
\mathbf{W}_{\mathbf{o}_{11}}, & \ldots, & \mathbf{W}_{\mathbf{o}_{\mathrm{pp}}}
\end{array}\right)
\end{aligned}
$$

with, for $i=1, \ldots, p$,

$$
\begin{aligned}
\mathbf{W}_{\mathbf{c}_{\mathbf{i i}}} & =\frac{\beta_{i i}}{4 \xi_{i} \omega_{i}} \mathbf{I}_{\mathbf{2}} \\
\mathbf{W}_{\mathbf{o}_{\mathbf{i i}}} & =\frac{\gamma_{i i}}{4 \xi_{i} \omega_{i}^{3}} \mathbf{I}_{\mathbf{2}}
\end{aligned}
$$

where $\beta_{i i}=\mathbf{b}_{\mathbf{i}} \mathbf{b}_{\mathbf{i}}^{t}, \gamma_{i i}=\mathbf{c}_{\mathbf{i}}^{t} \mathbf{c}_{\mathbf{i}}$ are the coefficients of modal gramians, and $\mathbf{I}_{\mathbf{2}}$ is the $2 \times 2$ identity matrix. For a given mode $\left(\xi_{i}, \omega_{i}\right), \beta_{i i}$ and $\gamma_{i i}$ scalars represent the relative influence of the different actuators on the $i^{t h}$ mode, and the way the $i^{t h}$ mode is seen through the different sensors. Let's note that these gramian approximation writings indicate that the high-damped and high-frequency modes are amongst the least controllable and observable ones.

\section{E. Computation of the balanced gramian}

In order to have a consistent level indicator of both controllability and observability of the modes of a system, it is not enough to look separately at its controllability and observability gramians. The balanced case, where controllability and observability gramians are diagonal and equal to Hankel singular values (HSV) gramian $\Gamma$,

$$
\mathbf{W}_{\mathbf{c}}=\mathbf{W}_{\mathbf{o}}=\boldsymbol{\Gamma}=\operatorname{diag}\left(\sigma_{i}\right)
$$

where $\sigma_{i}$ are the HSV of the system, is a useful tool for quantifying the joint controllability and observability of a system. It actually characterizes efficient outputs control by the inputs [43].

It is shown that, when the damping ratios decrease to zero, the balanced state coordinates are decoupled and coincide with the modal coordinates [43]. Indeed, HSV equal to the square root of the product of the controllability and observability modal coefficients of this mode, so that simple formulas expressing the asymptotic singular values as functions of the modal parameters can be derived :

$$
\sigma_{i}=\frac{\sqrt{\mathbf{c}_{\mathbf{i}}^{t} \mathbf{c}_{\mathbf{i}} \mathbf{b}_{\mathbf{i}} \mathbf{b}_{\mathbf{i}}^{t}}}{4 \xi_{i} \omega_{i}^{2}}
$$

HSV describe the degree that a given modal state contributes to the input/output energy flow through the system. 


\section{F. Formulation of new control-oriented criteria for the opti- mization of SISO flexible system}

In this section, we study new control-oriented criteria in the particular case of SISO systems. They will be applied for the optimization of a flexible piezoactuated microgripper afterwards. A single input voltage will actuate the piezoactive blocks of the device, and the mechanical end-effector port will be represented by a single output. One of the objectives of the optimization is to tune the open-loop transfer function to one of a reduced SISO model type, while forcing the system to have a collocated behaviour (by adjusting the minimum-phase properties). Finally, this will allow the easy implementation of Low Autorithy Control.

1) Evaluation of the model reduction cost for Low Authority Control: Resonance peaks amplitudes must be maximized in the frequencies bandwidth $\left[0, \omega_{c}\right]$ to increase authority control on these dominant modes. On the contrary, the amplitudes of resonance peaks after cut-off frequency must be minimized to increase gain margin and to limit modes destabilization in this area (spillover phenomenon) (Fig.6).

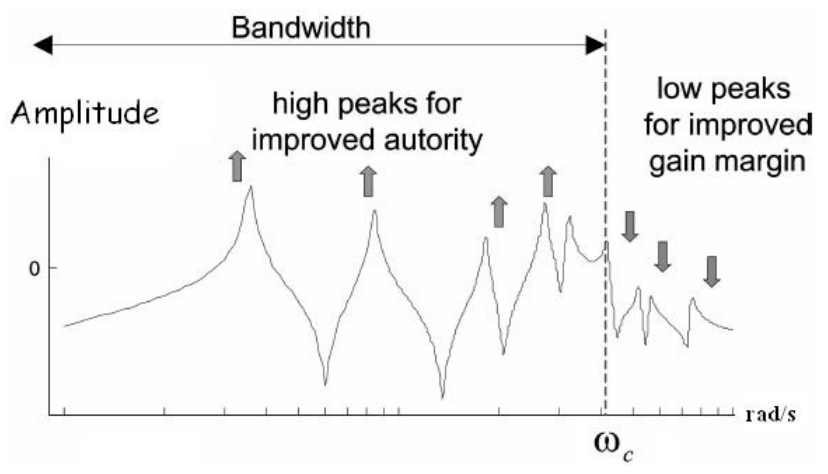

Fig. 6. Desired form of the open-loop FRF.

Thanks to their $H_{\infty}$ norm interpretation, HSV are simple metrics for quantifying the modal autority of the system. $H_{\infty}$ norm characterizes the maximal amplification of the input signal energy that the system can produce. In $M I M O$ case, it is equivalent to the maximum singular value of the system,

$$
\left\|\mathbf{G}_{\mathbf{i}}\right\|_{\infty}=\max _{\omega} \sigma_{\max }\left(\mathbf{G}_{\mathbf{i}}(\omega)\right)
$$

In $S I S O$ case, it simply represents the maximum amplitude value of the frequency response, formulated as follows for small damping system,

$$
\left\|G_{i}\right\|_{\infty} \simeq \frac{\left|c_{i} b_{i}\right|}{2 \xi_{i} \omega_{i}^{2}}
$$

so that, according to (25), it can be almost proportionally linked to the corresponding HSV of $G_{i}$ as follows:

$$
\left\|G_{i}\right\|_{\infty} \simeq 2 \sigma_{i}
$$

Thus, the $k$ first resonant modes (where $k<p$ ) will be optimized to guarantee high HSV compared to the ones out of the bandwidth. HSV of $G$ realization, as defined in (25), sorted in ascending order according to $\omega_{i}$ modes, are maximized for $i=1, \ldots, k$. The modal states with small HSV are both weakly controllable and weakly observable, and will be removed from the reduced-system.

As a consequence, the resulting dominant reduced-order model $G_{r}$ defined as

$$
G_{r}(j \omega)=\sum_{i=1}^{k} G_{i}(j \omega)
$$

will match the full model $G(j \omega)$ with an accuracy related to the size of the HSV which were discarded. The approximation error upper-bound for a balanced truncated model satisfies the inequality [44]:

$$
\left\|G(j \omega)-G_{r}(j \omega)\right\|_{\infty} \leq 2 \sum_{i=k+1}^{p} \sigma_{i}
$$

To improve simultaneously the control authority on the $k$ first dominant modes and the accuracy of the reduced order model, the first new criteria implemented in FlexIn is the following:

$$
J_{1}^{k}=\frac{\sum_{i=1}^{k} \sigma_{i}}{\sum_{i=k+1}^{p} \sigma_{i}}
$$

where the HSV are defined in their modal form by (25) for flexible structures. In our case study, an order $k=2$ is chosen as a good compromise for the piezoelectric flexible structure model. On the one hand, a reduction at low order facilitates the system identification and the regulator synthesis afterwards. On the other hand, the very simple case $k=1$ will restrain too much the number of candidate solutions for the global optimization.

2) Optimization of the required minimum-phase properties for collocated behaviour: The most useful characteristic of a collocated system is the interlacing of poles and zeros along the imaginary axis. For a lightly damped structure, poles and zeros are located in the left half-part in the pole-zero map (Fig.7). Such systems are minimum of phase. This results in a phase response that lies continuously between $0^{\circ}$ and $180^{\circ}$. In the case where the actuator and sensor are quasicollocated, this property is still valid in the low frequency spectrum. Collocated systems are known to possess interesting properties. Vibration control of flexible structures involving
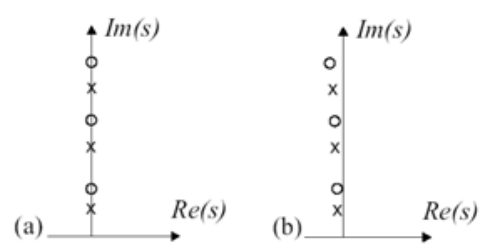

Fig. 7. Poles (x) and zeros (o) locations of a collocated system: (a) undamped, $(b)$ lightly damped (Figure is symmetric towards Real axis).

collocated characteristics was discussed in [45] and [46], among others. Control was shown to have simple stability criteria due to the alternating poles and zeros pattern. For 
example, one way of adding damping to the structure is to use Positive Position Feedback (PPF), as proposed in [47], which is stable in the presence of uncontrolled modes in the bandwidth, and which rolls off quickly at higher frequencies, reducing the risk of destabilizing systems with high-frequency dynamics. This makes collocation of the transfert function an attractive control approach.

In FlexIn, an evaluation function was implemented to be used in the optimization process in order to obtain systems designs with collocated type open-loop transfer function, forcing the resonances (poles) and antiresonances (zeros) alternating in the reduced model. Inspired by [40] and [46], it can be shown that the maximization of the following discrete criterion will imply the interlacing pole-zero pattern exhibited by a collocated transfer function:

$$
J_{2}^{k}=\left|\sum_{i=1}^{k} \operatorname{sign}\left(c_{i} b_{i}\right)\right|
$$

where $\operatorname{sign}()=+1,0,$.-1 , according to the argument sign. The sum over $i$ concerns all the modes contained in the frequency spectrum of the first $k$ dominant modes, where the alternative is desired. This criteria will force the static gains of $G_{i}$ in the spectrum of interest to have the same sign. (In our application case $k$ is set to 2 , and only two numerical values are possible: the maximum value is $J_{2}^{2}=2$, otherwise $J_{2}^{2}=0$.)

\section{Multiobjective Optimal SYNTHESIS OF A MONOLITHIC COMPLIANT PIEZOACTUATOR}

The concepts presented previously have been applied to the design of a microgripper actuator, considering a multi-criteria optimization problem, with both static mechanical (stroke and force at the output node of the structure) and control-oriented $J_{1}^{2}$ and $J_{2}^{2}$ fitnesses.

\section{A. Optimization problem specifications}

We consider the synthesis of a symmetric monolithic microactuation mechanism, made of a single piezoelectric material PIC151 from PI Piezo Ceramic Technology [48] (see TableI). Let us note that, since damping cannot be accurately known a priori before an identification procedure, modal damping is taken constant in the optimization algorithm, and equal to $1 \%$ for all resonant modes. (Thus, it excludes the influence of the damping ratio variations in $J_{1}^{2}$ criterion.)

At the end, the whole microactuator will be machined using Laser cutting technology into an electroded piezoelectric plate whose dimensions are indicated on (Fig.8). To take advantage of the maximum size allowed for the piezoactive structure, the half microactuator topology is considered to have a maximal size of $15 \mathrm{~mm} \times 9 \mathrm{~mm}$, and a constant thickness of $200 \mu \mathrm{m}$.

The half-microactuator topology is defined to be made of either passive or active blocks inside a $2 \times 2$ mesh (Fig.9). Actually, active blocks are those which will be bonded with electrodes, exploiting the inverse piezoelectric effect, while passive blocks will be made in the same piezoelectric material but without electrodes. For the optimal synthesis run, the number of active blocks in the half-part will be allowed to
TABLE I

ELECTROMECHANICAL CHARACTERISTICS OF PIEZOELECTRIC MATERIAL PIC151 CONSIDERED IN THE OPTIMIZATION.

\begin{tabular}{ll}
\hline$\overline{\text { Young modulus }(Y)}$ & $66,667 \mathrm{MPa}$ \\
\hline Poisson ratio $(\nu)$ & 0.3 \\
\hline Coupling piezoelectric coefficient $\left(d_{31}\right)$ & $-210 \times 10^{-12} \mathrm{~m} . \mathrm{V}^{-1}$ \\
\hline Density $(\rho)$ & $7800 \mathrm{~kg} \cdot \mathrm{m}^{-3}$ \\
\hline Modal damping ratio $\left(\xi_{i}\right)$ & $1 \%$ \\
\hline \hline
\end{tabular}

vary between 1 and 4 . When external voltages are applied to the blocks electrodes, the output node of the structure has to move along the $x$-axis and to produce a gripping force. For evaluation of static mechanical criteria, the potential difference between upper and lower face is taken equal to $200 \mathrm{~V}$. The size ratio of the blocks can vary as $b_{\max } / b_{\min } \in \llbracket 1 ; 2 \rrbracket$ and $a_{\max } / a_{\min } \in \llbracket 1 ; 2 \rrbracket$ (see Fig.9 for details about $a$ and $b$ parameters definitions). The number of blocked nodes is comprised between 1 and 3 among the locations permitted which are reported on Fig.9.

Finally, the four objective functions to be maximized simultaneously with FlexIn are: output free stroke $\delta_{x}$, blocking force $F_{x}$ (i.e. gripping force in our application), $J_{1}^{2}$ and $J_{2}^{2}$.

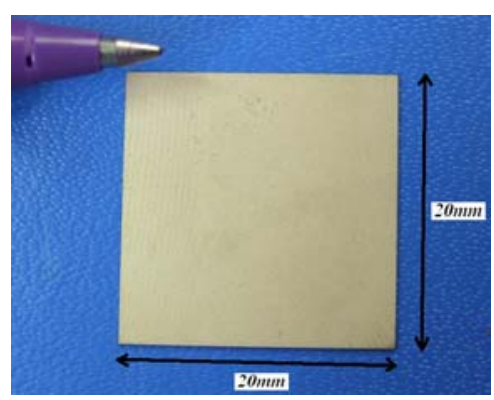

Fig. 8. Electroded PIC 151 plate with its dimensions to be machined using Laser cutting technology (thickness: $e_{p}=200 \mu \mathrm{m}$ ). Electrodes are deposited on the whole upper and lower surfaces.

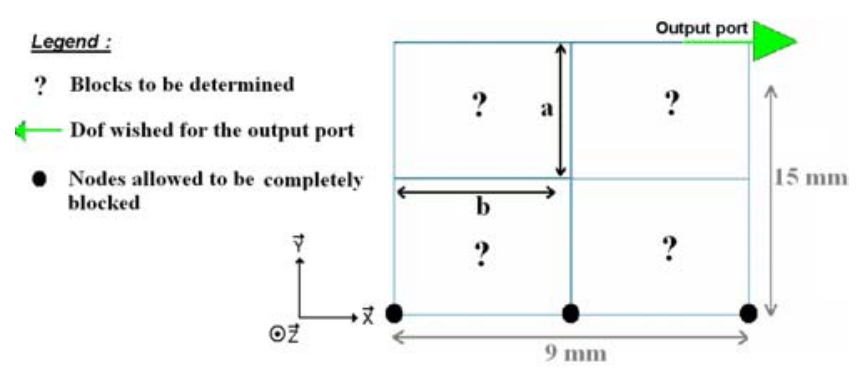

Fig. 9. Mesh of the left-part of the symmetric PZT compliant micro-actuator with imposed and permitted boundary conditions. $a$ and $b$ optimization parameters define the relative height and width of the blocks.

\section{B. Optimal synthesis results}

FlexIn method can generate efficient piezoelectric actuated flexible mechanisms for microgripper devices. The best compromise structures are kept, when the genetic algorithm does not find any new pseudo-optimum during 130 subsequent 
generations. The set of pseudo-optimal solutions can be represented on Pareto fronts, giving their different fitness performances along each other (Fig.10). The designer can choose among these solutions. Let us note that lots of individuals have the same $J_{1}^{2}$ and $J_{2}^{2}$ values, but they differ when considering force and displacement performances.
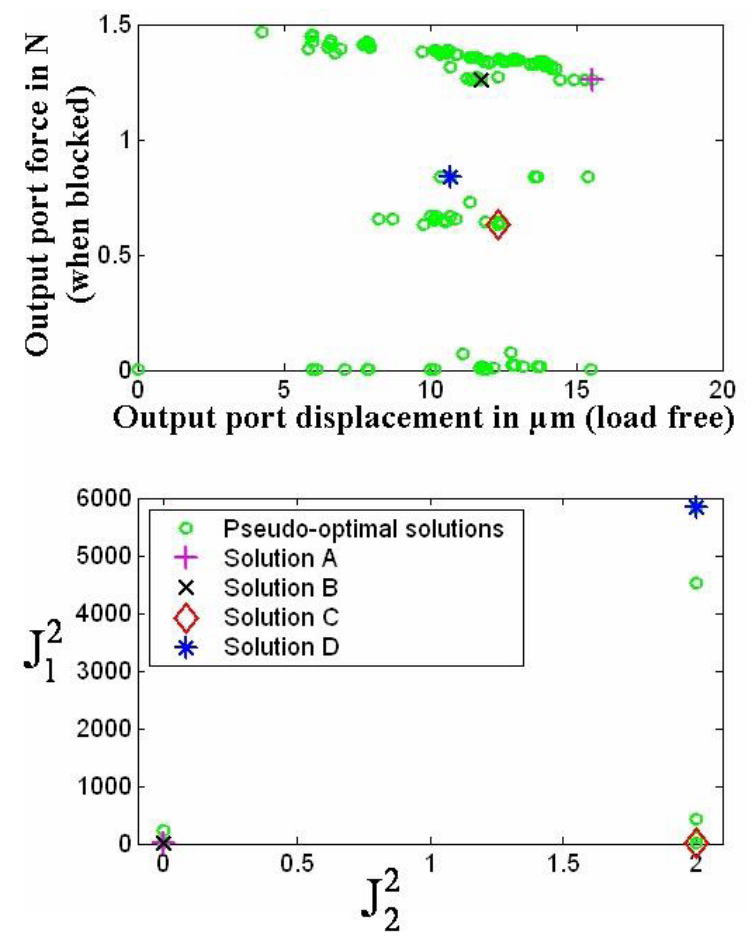

Fig. 10. Pareto fronts of compliant mechanisms synthesized using FlexIn (genetic parameters used: population of 100 individuals, mutation probability of $45 \%$ on genes and $60 \%$ on individuals), and four chosen pseudo-optimal solutions $(A, B, C$ and $D)$.

From these fronts, four chosen pseudo-optimal solutions, whose topologies are presented on Fig.11 and referred as $A, B, C$ and $D$ structures in the following, will illustrate performance comparison (see TableII). Amongst the Pareto set of solutions, these four structures have been chosen, because they exhibit good quasi-static performances (high stroke $\delta_{x}$ and blocking force $F_{x}$ values). Moreover, their openloop transfer behaviors differ from each other according to their respective control-oriented criteria values $J_{1}^{2}$ and $J_{2}^{2}$. The compared analysis of these four structures will help to illustrate the $J_{1}^{2}$ and $J_{2}^{2}$ link to the shape of their Frequency Response Functions.

- Structure $A$ has bad control-oriented criteria performances. Firstly, it exhibits non-minimum phase transfer in the low frequency spectrum $\left(J_{2}^{2}=0\right)$, since two antiresonances occur between the two first resonances. Then, the authority control on the two first resonant modes is poor compared to other modes, particularly to the fourth mode. Resulting Frequency Response Function is reported on Fig. 12.

- Structure $B$ presents a good $J_{1}^{2}$ criterion performance: the authority control on the two first resonant modes is well optimized, resulting in an important roll-off after the second resonance. Unfortunately, such a structure also exhibits non-minimum phase transfer in that low frequency spectrum $\left(J_{2}^{2}=0\right)$, as shown on Fig.13.

- As expected with $J_{2}^{2}=2$, structure $C$ is a minimum phase system in the spectrum of interest. Nevertheless, as expected by its poor $J_{1}^{2}$ criteria, some high frequency resonant modes are as dominant as the first low frequency ones. For example, on Fig.14, amplitude of the fourth resonance has the same magnitude as the first one, so that some high frequency modes cannot be neglected in the model reduction step.

- Structure $D$ is an example of structure with both good $J_{1}^{2}$ and $J_{2}^{2}$ control-oriented criteria. Its frequency response function shape is the one intended (Fig.15).

TABLE II

Performances Criteria values of the $A, B, C$ And $D$ PSEUDO-OPTIMAL COMPLIANT PIEZOACTUATORS SYNTHESIZED BY FLEXIN.

\begin{tabular}{ccccc}
\hline \hline $\begin{array}{c}\text { Selected } \\
\text { solutions }\end{array}$ & $\delta_{x}$ & $F_{x}$ & $J_{1}^{2}$ & $J_{2}^{2}$ \\
\hline $\mathbf{A}$ & $15.55 \mu \mathrm{m}$ & $1.26 N$ & 2.24 & 0 \\
$\mathbf{B}$ & $11.74 \mu \mathrm{m}$ & $1.26 N$ & 21.00 & 0 \\
$\mathbf{C}$ & $12.34 \mu \mathrm{m}$ & $0.63 N$ & 0.28 & 2 \\
$\mathbf{D}$ & $10.69 \mu \mathrm{m}$ & $0.84 N$ & 5842.35 & 2 \\
\hline \hline
\end{tabular}
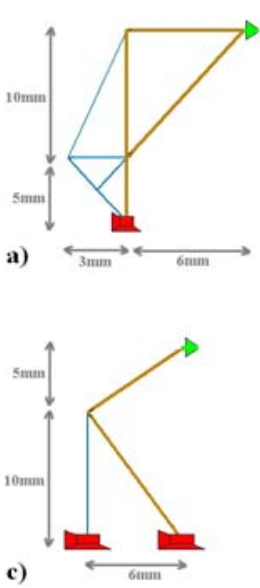

b)
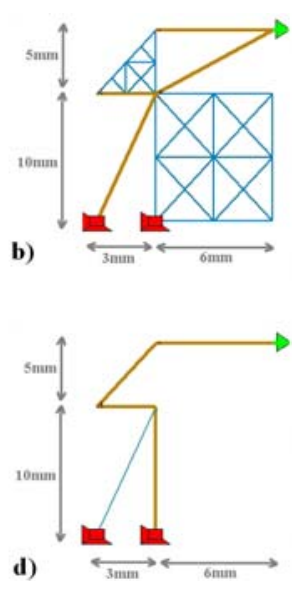

Fig. 11. FlexIn representations of the four pseudo-optimal selected piezoactuators (bold yellow lines refer to piezoactuated blocks, whereas fine blue lines refer to piezo-passive ones).

As regard to its very interesting mechanical and controloriented performances, we chose to focus on the study of structure $D$ in the following.

\section{Validation of structure $D$}

The results obtained using the 2D FE model implemented in FlexIn have been compared and confirmed by a commercial 3D multiphysics FE software (fine tetrahedric mesh).

The active beams are supplied by $V= \pm 100 \mathrm{~V}$, which results in the desired deformed shape (Fig.16). The values of $x$-displacement at the output port is $9.23 \mu \mathrm{m}$ (about $14 \%$ relative error compared to FlexIn calculation), demonstrating 

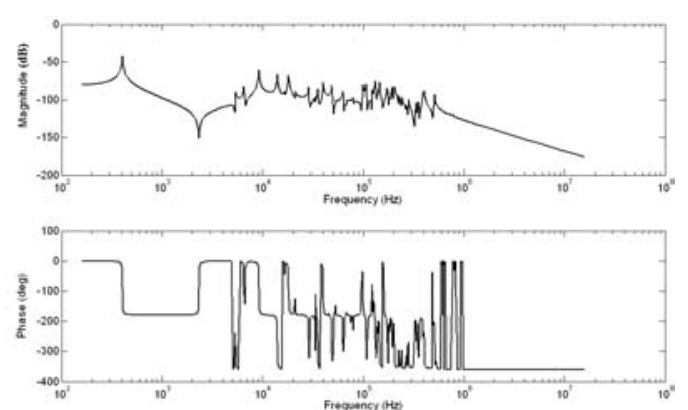

Fig. 12. Bode diagram of structure A between input (voltage $\mathrm{u}$, in $V$ ) and output (deflexion $\delta_{x}$, in $\mu m$ ) simulated by FlexIn.
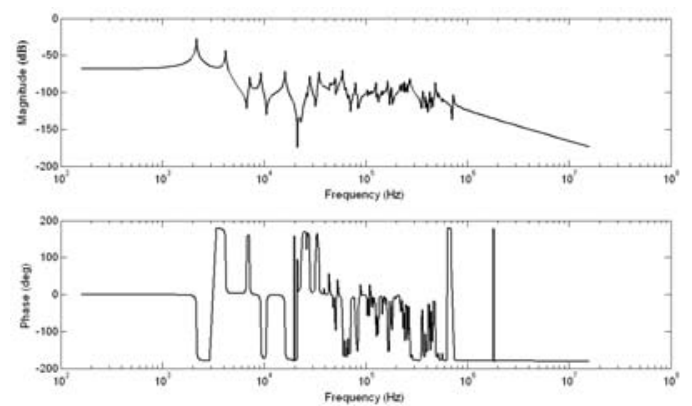

Fig. 13. Bode diagram of structure B between input (voltage $\mathrm{u}$, in $V$ ) and output (deflexion $\delta_{x}$, in $\mu m$ ) simulated by FlexIn.
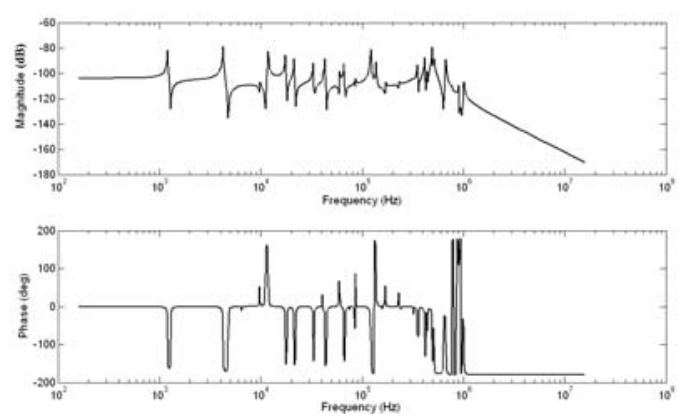

Fig. 14. Bode diagram of structure $\mathrm{C}$ between input (voltage $\mathrm{u}$, in $V$ ) and output (deflexion $\delta_{x}$, in $\mu m$ ) simulated by FlexIn.
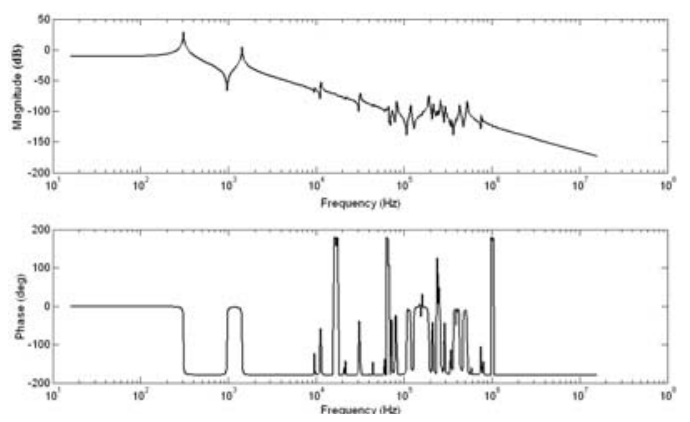

Fig. 15. Bode diagram of structure $\mathrm{D}$ between input (voltage $\mathrm{u}$, in $V$ ) and output (deflexion $\delta_{x}$, in $\mu m$ ) simulated by FlexIn. the nearly good accuracy in the static case of our preliminary design method. This difference can be explained by the simplifying assumption made in FlexIn: the 2D FE model (with only one element per elementary beam) does not take into account the $3 \mathrm{D}$ edge effects at the beams connections, which can be of importance for multi-beams structures.

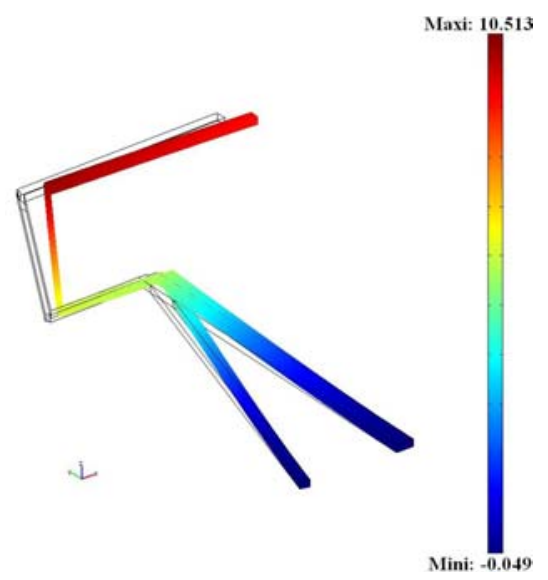

Fig. 16. $3 D$ simulation of the $x$-displacement (in $\mu m$ ) when the half piezoactuated structure is activated under $\pm 100 \mathrm{~V}$, and associated deformed shape using the $3 D$ FE Software.

Dynamic behaviour of the device has also been plotted using the $3 D$ FE Software (Fig.17). Let us note that this step is of great importance for post-treatment procedure: indeed, as the 2D FlexIn method can not model out-of-plane 3D resonant modes, such as twist modes, they could not be taken into account in the preliminary frequency response optimization of the device. As shown on Fig.17, 2D FRF shape of structure $D$ is nearly unmodified by adding the resonant $3 \mathrm{D}$ effects in the low frequency spectrum of interest. In particular, the property of minimum-phase system, and the good modal control authority on the two first resonant modes are still maintained. As shown in TableIII, the low shifts of natural frequencies are very acceptable, and prove the good accuracy of the method in the dynamic case as well.
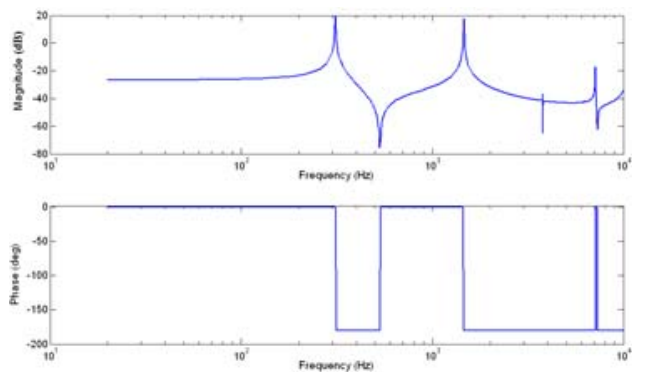

Fig. 17. Bode diagram of structure $D$ between input voltage and output deflexion $\delta_{x}$ in $\mu m$ (using $3 D$ FE Software simulation for undamped model).

\section{EXPERIMENTAL VALIDATION STUDY OF THE MONOLITHIC COMPLIANT PIEZOACTUATOR $D$}

After $3 D$ FE simulation and validation, piezoactuated structure $D$ has been prototyped and experimentally tested. 
TABLE III

COMPARISONS OF THE FIRST TWO NATURAL FREQUENCIES ESTIMATIONS USING $2 D$ FLEXIN AND 3D FE SOFTWARES.

\begin{tabular}{ccc}
\hline \hline FlexIn & FE Software & Relative error \\
\hline $306.23 \mathrm{~Hz}$ & $310.56 \mathrm{~Hz}$ & $1.4 \%$ \\
$1429.43 \mathrm{~Hz}$ & $1458.48 \mathrm{~Hz}$ & $2.0 \%$ \\
\hline \hline
\end{tabular}

\section{A. Fabrication of the monolithic compliant piezoactuator}

The whole device has been machined in an electroded PIC151 plaque (Fig.8) by a laser cutting process. PZT is a ceramic material which can be easily reshaped by laser cutting without perceptible loss of its piezoelectric and material properties.

- At its maximum output power, laser is capable of precisely cutting through PZT, quickly enough not to overheat the sample.

- By reducing the power of the laser beam, it is also possible to pattern the very thin layer of gold-nickel that constitutes the electrode layer of the piezoelectric plate.

Fig.18 shows the device $3 D$ model with top electrode patterns. The whole structure is divided into an active (electroded) and a passive areas, which both will be free to bend, and the base remaining area which will be clamped and kept out from bending (boundary conditions of fixed nodes). The clamping area is where the electric wires will feed the electrodes, respectively with $V_{\text {left }}$ and $V_{\text {right }}$ for the actuated left and right arms of the gripper. (Fig.19 shows a photo of the machined piezoelectric layer.)

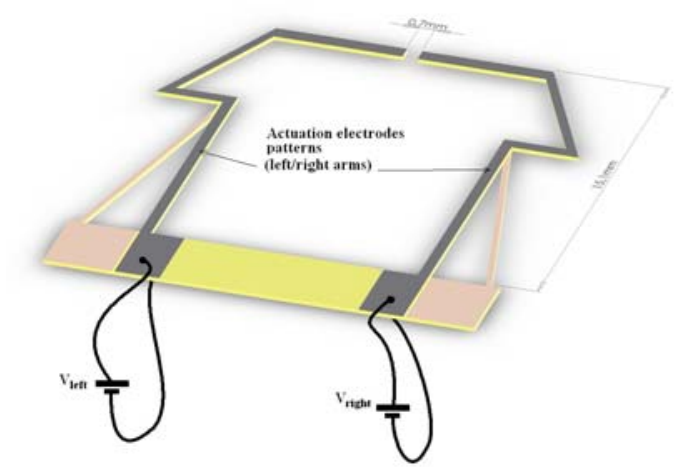

Fig. 18. $3 D$ CAD model of the piezoeletric device with top face electrode patterns. $V_{\text {left }}$ (resp. $V_{\text {right }}$ ) is the controlled input for actuating the left (resp. right) arm.

\section{B. Experimental characterizations of the prototype and vali- dation}

The microactuator prototype is clamped, and placed on $x-y$ $z$ micropositioning linear stages, which are manually operated (Fig.20). The piezoelectric actuator requires high voltage to provide adequate deflection. Thus, the device is connected to a linear power amplifier, with an amplification ratio of fifty, linked to the spectrum analyser source. Output displacement at the tip of the piezoelectric structure is measured along $x$ axis using a $0.1 \mu \mathrm{m}$-resolution laser sensor. The analog ouput

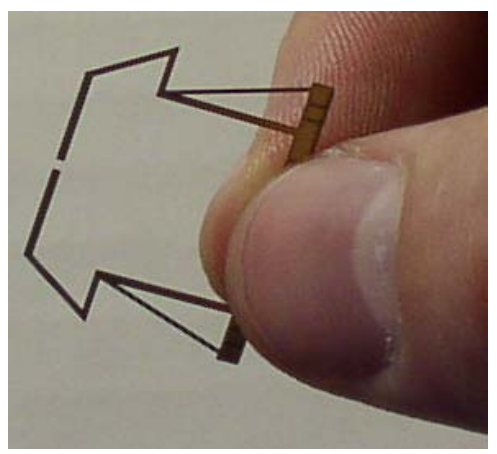

Fig. 19. Photo of the prototyped piezoelectric monolithic device, obtained by laser cutting.

of the laser is directly connected to the spectrum analyser, and the response is recorded for subsequent analyses.

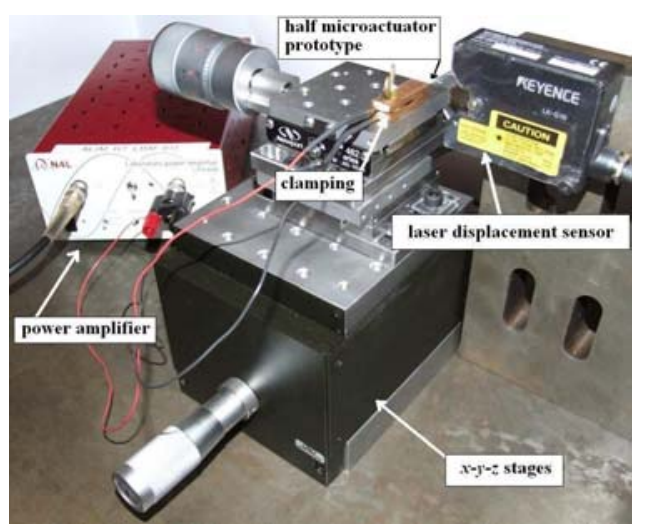

Fig. 20. Experimental setup.

In static loading, experimental $x$-displacement has been measured to $9.4 \mu \mathrm{m}$ under $\pm 100 \mathrm{~V}$ voltage activation, which is close to the predicted simulation results $9.37 \mu \mathrm{m}$. Experimental frequency response data are reported on Fig.21. It appears that the minimum-phase property is still kept into the desired spectrum (until the $2^{\text {nd }}$ resonance frequency). However, although the third resonance was not expected with such a high amplitude, identification process has been still performed considering the two first dominant modes involved in the reduced model, as follow:

$$
G_{r}(j \omega)=\frac{K_{1}}{\omega_{1}^{2}-\omega^{2}+2 j \xi_{1} \omega_{1} \omega}+\frac{K_{2}}{\omega_{2}^{2}-\omega^{2}+2 j \xi_{2} \omega_{2} \omega}
$$

Identified characteristics of $G_{1}$ and $G_{2}$ transfers are mentionned in Table.IV. (Let us note that $\xi_{1} \neq \xi_{2}$ in experimentation, contrary to the prior hypothesis used for the FlexIn simulation.) The phase is slightly rolling off, (see Fig.21) coming from the bandwidth limitation of the laser sensor, whose cutoff frequency is about $1 \mathrm{KHz}$. Due to mounting imprecisions of the experimental set-up, we observe extra-resonance modes (third and forth peaks) which in fact correspond to out-ofplane deflection of the tip. They were not predicted by simulations because FlexIn uses a planar model. In our preliminary electromechanical model implemented in FlexIn, nonlinear behavior of the piezoelectric material, especially hysteresis, 

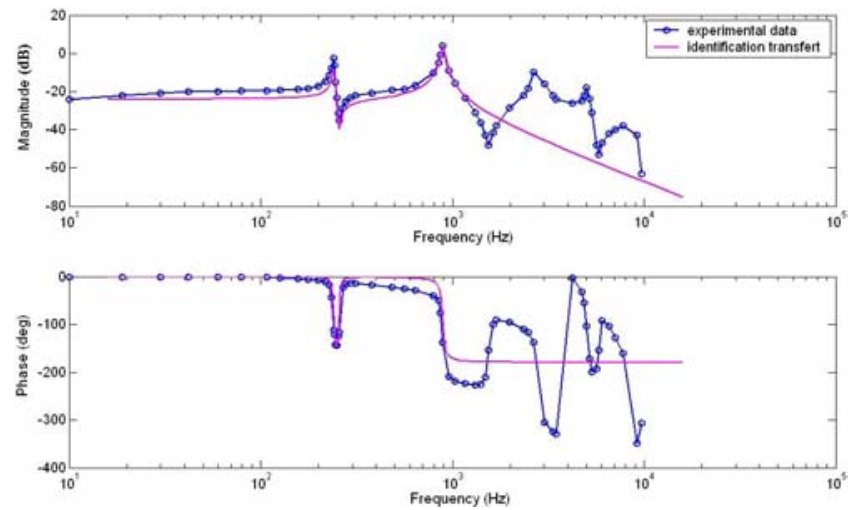

Fig. 21. Experimental frequency transfer function of the piezoactuato between input (voltage $\mathrm{u}$, in $V$ ) and output (deflexion $\delta_{x}$, in $\mu m$ ), and identified transfer function of the reduced-model $G_{r}=G_{1}+G_{2}$

TABLE IV

IDENTIFIED PARAMETERS OF $G_{1}$ AND $G_{2}$ TRANSFER FUNCTIONS.

\begin{tabular}{ccc|ccc}
\hline \hline \multicolumn{2}{c|}{$G_{1}$} & \multicolumn{3}{c}{$G_{2}$} \\
\hline$K_{1}$ & $\xi_{1}$ & $\omega_{n 1}($ rad.s & \\
\hline 0.87 & $1.2 \%$ & $1.51 \times 10^{3}$ & $K_{2}$ & $\xi_{2}$ & $\omega_{n 2}\left(\right.$ rad.s $\left.{ }^{-1}\right)$ \\
\hline \hline
\end{tabular}

has not been taken into account. Thus, the hysteresis curve of the piezoelectric device endpoint displacement controlled by voltage actuation has been measured, and is shown with the linear model fit on Fig.22. Design of robust closed-loop actuator control will therefore be useful to treat such nonlinearity. This is also a near perspective of our research work. An other perspective would consist in driving the piezoelectric device using a charge source as mentionned in [49]. A last method would consist in using inverse hysteresis model (the most common one being Preisach technique) in a feedforward linearize loop control [50].

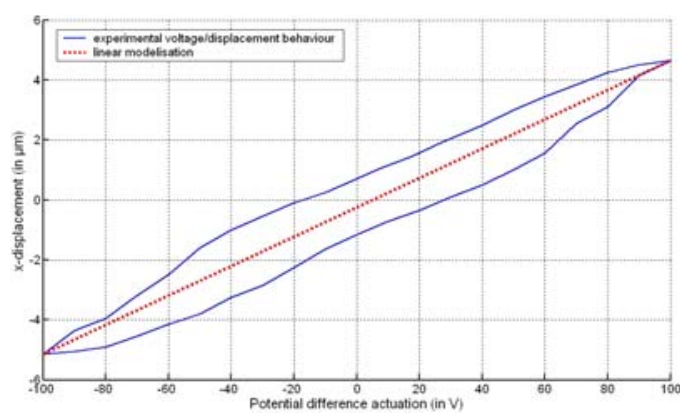

Fig. 22. Measured quasi-static relationship between applied voltage actuation (in $V$ ) and endpoint displacement $\delta_{x}$ (in $\mu m$ ).

\section{CONCLUSIONS}

A new concept of optimal design method for smart compliant mechanisms has been presented. This method, called FlexIn, can consider a smart compliant mechanism as an assembly of passive and active compliant building blocks made of PZT, so that actuators are really integrated in the structure.
Complex multi-objective design problems can be solved by FlexIn, taking advantage of versatile criteria to synthesize high performance microrobotic flexible mechanisms designs. In addition to classical mechanical criteria, currently encountered in topology optimization (i.e. force and displacement maximization), FlexIn considers now simultaneously efficient control-based criteria.

Each FlexIn synthesized structure being described by its own modal state representation, from the first designing step, specific control requirements can be optimized.It can be of great interest for Low Authority Control of flexible devices. Open-loop transfer considerations lead to two new efficient numerical criteria. A first criterion takes advantage of the specific expressions of the balanced gramian eigenvalues of a flexible structure, to modulate resonance amplitudes of its frequency response function in a spectrum of interest. A second criterion efficiently forces minimum-phase system property. These two criteria, coupled with mechanical ones, help designing non-intuitive compliant mechanisms, to meet specific mechanical and active control requirements.

This optimization strategy was tested for the optimal design of a microgripper actuator. The results obtained have proved that the method can furnish innovative and efficient solutions, very different from well-known actuation schemes such as unimorph or bimorph PZT actuators, which are widespread in the design of microrobotic manipulators. Post-treatment FE simulations confirm the accuracy of FlexIn method estimations, in both static and dynamic cases.

A prototype of an optimal flexible piezoactuated device has been successfully machined by Laser cutting process. Finally, experimental tests proved that the design optimization process outlined in this research work is valid. FlexIn is a great tool to design compliant systems for successful operations in micromanipulation tasks, and many others.

Future research includes optimal combination of sensors and actuators into the structure. A perspective is to take advantage of the direct piezoelectric effect, to consider as well force sensor integration inside monolithic piezoelectric structures to synthesize adaptronic devices.

\section{ACKNOWLEDGMENT}

Particular thanks to Dr. Gerard Michel, from the 'Ecole Nationale Supérieure de Mécanique et des Microtechniques' (ENSMM) in Besançon (France), for helping us to machine prototypes of the piezoactuator, and to Ing. Pascal Chambaud, from the CEA List in Fontenay-aux-Roses, for helping setting up the experimental characterization platform.

\section{REFERENCES}

[1] Lee W.H., Kang B.H., Oh Y.S., Stephanou H., Sanderson A.C., Skidmore G., Ellis M., "Micropeg manipulation with a compliant microgripper", in Proc. IEEE Int. Conf. on Robotics and Automation, Taipei, Taiwan, pp. 3213-3218, September 2003

[2] Chang H.C., Tsai J.M.L., Tsai H.C., Fang W., "Design, fabrication, and testing of a 3-DOF HARM micromanipulator on (111) silicon substrate", Sensors and Actuators, vol. 125, pp. 438-445, 2006.

[3] Kota S., Ananthasuresh G.K., Crary S.B., and Wise K. D., "Design and fabrication of micro-electromechanical systems", ASME Journal of Mechanical Design, vol. 116, pp. 1081-1088, 1994. 
[4] Saggere L., Kota S., "Static shape control of smart structures using compliant mechanisms", AIAA Journal, vol. 37, pp. 572-578, 1999

[5] Kim D.H., Lee M.G., Kim B., Sun Y., "A superelastic alloy microgripper with embedded electromagnetic actuators and piezoelectric force sensors: a numerical and experimental study", Smart Materials and Structures, vol. 14, pp.1265-1272, 2005.

[6] Frecker M., Haluck R., "Design of a multifunctional compliant instrument for minimally invasive surgery", Journal of Biomedical Engineering, vol 127, pp. 990-993, November 2005.

[7] Houston K., Sieber A., Eder C., Tonet O., Menciassi A., Dario P., "Novel Haptic Tool and Input Device for Real Time Bilateral Biomanipulation addressing Endoscopic Surgery ", Proc. of the 29th Annual International Conference of the IEEE EMBS, Lyon, France, August 23-26, pp. 198201, 2007.

[8] Breguet J.M., and al., "Monolithic piezoceramic flexible structure for micromanipulation", 9th International Precision Engineering Seminar and 4th International Conference on Ultraprecision in Manufacturing Engineering, pp. 397-400, Braunschweig Germany, 1997.

[9] Agnus J., Nectoux P. , Chaillet N., "Overview of microgrippers and micromanipulation station based on a MMOC microgripper", Proc. of the IEEE International Symposium on Computational Intelligence in Robotics and Automation, pp. 117-123, 2005.

[10] Frecker M., Canfield S., "Optimal design and experimental validation of compliant mechanical amplifiers for piezoceramic stack actuators", Journal of Intelligent Material Systems and Structures, vol. 11, pp. 360$369,2000$.

[11] Kota S., "Tailoring unconventional actuators using compliant transmissions : design methods and applications", IEEE/ASME Transactions on Mechatronics, vol. 4, pp. 396-408, December 1999.

[12] Lau G. K., and al., "Systematic design of displacement - amplifying mechanisms for piezoelectric stacked actuators using topology optimization", Journal of Intelligent Material Systems and Structures, vol. 3985, pp. 583-591, 2000.

[13] Barboni R., and al., "Optimal placement of PZT actuators for the contro of beam dynamics", Smart Material and Structures, pp. 110-120, 2000

[14] Maddisetty H., Frecker M., "Dynamic topology optimization of compliant mechanisms and piezoceramic actuators", ASME Journal of Mechanical Design, vol. 126, pp. 975-983, 2002.

[15] Abdalla M., and al., "Design of a piezoelectric actuator and compliant mechanism combination for maximum energy efficiency", Smart Material and Structures, vol. 14, pp. 1421-1430, 2005.

[16] Nelli Silva E.C., Kikuchi N., "Design of piezoelectric transducers using topology optimization", Smart Material and Structures, vol. 8, pp. 350 -365 , USA, 1999.

[17] Fablbusch S., and al., "Flexible Microrobotic System MINIMAN Design, Actuation Principle and control", Proc. of the IEEE/ASME International Conference on Advanced Intelligent Mechatronics, pp. 156161, Atlanta, Georgia USA, 1999.

[18] Du H., and al., "Topological optimization of mechanical amplifiers for piezoelectric actuators under dynamic motion", Smart Material and Structures, vol. 9, pp. 788-800, 2000.

[19] Frecker M., "Recent advances in optimization of smart structures and actuators", Journal of Intelligent Material Systems and Structures, vol.14 pp. 207-216, April/May 2003.

[20] Jonckheere E. A., "Principal components analysis of flexible systems Open-loop case", IEEE Trans. on Automatic Control, vol. 29, pp. 10951097, 1984

[21] Kermani M. R., Moallem M., Patel R. V., "Optimizing the performance of piezoelectric actuators for active vibration control", in Proc. of IEEE International Conference on Robotics and Automation, Washington DC, pp. 2375-2380, 2002.

[22] Chen W., and al., "Optimal sensor design and control of piezoelectric laminate beams", IEEE Trans. on Control Systems and Technology, vol. 12, pp. 148-155, 2004

[23] Collet M., "Shape optimization of piezoelectric sensors dealing with spill-over instability", IEEE Trans. on Control Systems Technology, vol. 9, pp. 654-662, 2001

[24] Kermani M. R., Patel R. V., Moallem M., "Flexure control using piezostack actuators: design and implementation", IEEE/ASME Trans. on Mechatronics, vol. 10, pp. 181-188, 2005.

[25] Liu W., Hou Z., Demetriou M. A., "A computational scheme for the optimal sensor/actuator placement of flexible structures using spatial $\mathrm{H} 2$ measures", Mechanical Systems and Signal Processing, vol. 20, pp. 881$895,2006$.

[26] Grossard M., Rotinat-Libersa C., Chaillet N., "Gramian-based optimal design of a dynamic stroke amplifier compliant micro-mechanism",
IEEE/RSJ International Conference on Robots and Systems, San Diego, USA, 2007

[27] Bernardoni P., and al., "A new compliant mechanism design methodology based on flexible building blocks", Smart Material and Structures, vol. 5383, pp. 244-254, USA, 2004.

[28] Bernardoni P., "Outils et méthodes de conception de structures mécaniques à déformations réparties et actionnement discret - applications en microrobotique" , PhD Thesis realized at the CEA, University Paris 6, France, 2004

[29] Grossard M., Rotinat-Libersa C., Chaillet N., "Redesign of the MMOC microgripper piezoactuator using a new topological method", IEEE/ASME International Conference on Advanced Intelligent Mechatronics, Zürich, Switzerland, 2007.

[30] Rotinat-Libersa C., Perrot Y., Friconneau J.-P., "Potentialities of optimal design methods and associated numerical tools for the development of new micro- and nano- intelligent systems based on structural compliance - An example -", IARP- IEEE/RAS-EURON JointWorkshop on Micro and Nano Robotics, Paris, France, 2006.

[31] Grossard M., Rotinat-Libersa C., Chaillet N., Perrot Y., "Flexible building blocks method for the optimal design of compliant mechanisms using piezoelectric material", 12th IFToMM World Congress, Besançon, France, 2007.

[32] Deb K., and al., "A fast elitist non-dominated sorting genetic algothm for multi-objective optimization: Nsga-II", Proc. of the 6th International Conference on Parallel Problem Solving from Nature, pp. 849-858, France, 2000.

[33] ANSI/IEEE Std. 176-1987 IEEE Standard on piezoelectricity, 1987.

[34] Hagood N. W., Chung W. H., Von Flotow A., "Modelling of piezoelectric actuator dynamics for active structural control", Journal of Intelligent Material Systems and Structures, vol. 1, pp. 327-354, 1990.

[35] Preumont A., "Mechatronics: Dynamics of Electromechanical and Piezoelectric Systems (Solid Mechanics and its Applications)", published by Springer, September, 2006.

[36] Moore B.C., "Principal component analysis in linear systems: controllability, observability, and model reduction", IEEE Transactions on Automatic Control, vol. 26, 1981.

[37] Abreu G. L. C., Ribeiro M., Steffen J. F., "Experiments on optimal vibration control of a flexible beam containing piezoelectric sensors and actuators", Journal of Shock and Vibration, vol. 10, pp. 283-300, 2003.

[38] Halim D., Moheimani S. O. R., "Experimental implementation of spatial $H_{\infty}$ control on a piezoelectric laminate beam", IEEE/ASME Transactions on Mechatronics, vol. 4, pp. 346-356, 2002.

[39] Halim D., Moheimani S. O. R., "Spatial $\mathrm{H}_{2}$ control of a piezoelectric laminate beam: experimental implementation", IEEE Transactions on Control System Technology, vol. 10, pp. 533-546, 2002.

[40] Aphale S. S., Fleming A.J., Moheimani S. O. R., "Integral resonant control of collocated smart structures", Smart Materials and Structures, vol.16, pp. 439-446, 2007.

[41] Lim K. B., Gawronski W., "Actuators and sensor placement for control of flexible structures", in Control and Dynamics Systems: Advances in Theory and Applications, ed. London, Academic Press, 1993.

[42] Hac A., Liu L., "Sensor and actuator location in motion control of flexible structures", Journal of Sound and Vibrations, vol. 167, pp. 239$261,1993$.

[43] Gawronski W., Lim K. B., "Balanced control of Flexible structures", Springer, London, 1996.

[44] Glover K., "All optimal Hankel-norm approximations of linear multivariable systems and their $L^{\infty}$-error bounds", International Journal of Control, vol. 39, pp. 1115-1193, 1984.

[45] Gevarter W. B., "Basic relations for control of flexible vehicles", AIAA Journal, vol. 8, pp. 666-672, 1970.

[46] Martin G. D. "On the control of flexible mechanical systems", $\mathrm{PhD}$ Dissertation, Stanford University, USA, 1978

[47] Fanson J. L., Caughey T. K., "Positive position feedback-control for large space structures", AIAA Journal, vol. 28, pp. 717-724, 1990.

[48] PI Piezo Ceramic Technology, Available: http://www.piceramic.com/, 2005.

[49] Main J. A., Garcia E., Newton D. V., "Precision position control of piezoelectric actuators using charge feedback", Journal of guidance, Control and Dynamics, vol. 18, pp. 1068-1073, 1995.

[50] Ge P., Jouaneh M., "Generalised preisach model for hysteresis nonlinearity of piezoelectric actuators", Precision Engineering, vol. 20, pp. 99-111, 1997. 